\section{Pre-enfermedad y puntos de corte, cuestionados}

\section{Pre-illness and cutting -points, terms begins questioned}

Se ha introducido al uso frecuente un neologismo "preenfermedad", en el ámbito de salud y de la población en general, supuestamente acusa mejor a la enfermedad o condición para prevenirla. Sin embargo, hay razones para considerarla como otro dislate de la medicina". Los "puntos de corte"' son acotaciones necesarias recomendables para reconocer individuos portadores de riesgos, dentro de la población. El uso de estos términos se ha convertido en abuso, sea porque es innecesario o porque se le usa fuera de contexto, dando lugar a temas de discernimientos que obstaculizan la armonía y el buen entendimiento en la prevención.

El artículo publicado por el Dr. Guillermo Alberto Pérez Fernández con el título "La arbitrariedad de los puntos de corte. Una reflexión desde la perspectiva de la preenfermedad" toca el tema de incumbencia en medicina preventiva, clínica y epidemiológica. Señala lo negativo de calificar como "pre-enfermedad" a quien, con el criterio "puntos de corte"' es detectado en campañas de prevención. Las observaciones de este tipo son bienvenidas con la idea de mejorar la calidad y seguridad de las acciones médicas en el nivel operativo, pues afecta la confiabilidad y métodos de observación epidemiológicos, programas preventivos y la relación médico-paciente.

Es natural que al incluir los recientes avances de la medicina en las acciones clínicas, la atención se concentre a ver sólo beneficios, y llaman la atención advertir inconvenientes no esperados. Las observaciones reportadas en dicho artículo deben servir, por lo menos, para alertar de algo anómalo a ser revisado. Consecuentemente, en el análisis valen las consideraciones como parte de una opinión.

El tema no es nuevo ${ }^{2}$, data por lo menos de la primera mitad del siglo $X X^{3}$. Ha sido trabajado con los estudios epidemiológicos prospectivos para buscar la asociación estadística entre los elementos sospechosos, seguidos luego por enfermedades crónicas. Finalmente se ha aceptado que sí hay relación de causa a efecto, pero se debe aclarar que no es conforme al conocido concepto de identidad, sino del renovado concepto de probabilidad, no fácil de comprender en el uso cotidiano y quizás contribuya a desacuerdos. Sin embargo, se acepta como guía de una duda cuantificada, para inferir riesgos y fundamentar la medicina preventiva y predictiva, en beneficio de la duda.

El asunto también ha sido revisado a la luz de las tablas de la verdad, para robustecer los criterios de validez y confiabilidad diagnóstica en las pruebas de escrutinio ${ }^{4}$. Gracias a ello, se han podido descubrir y rechazar casos, pero eso sí, no se puede desligar de posibles falsos positivos y falsos negativos, los cuales son parte del resultado, de la explicación y no de la solución.

No obstante, que las acciones de programas preventivos tengan un buen fundamento, cuentan con reconocimientos internacionales para el control de las enfermedades no transmisibles (ENT) o crónicas, entre las cuales se significan las enfermedades cardiovasculares, hay necesidad de atender los problemas emergentes inherentes a las acciones prácticas, especialmente porque en ellas es obligado el apoyo no médico, y éste pudiera no estar suficientemente adiestrado en el manejo de términos y conceptos vertidos en las normas que los rigen, por lo que surgen cuestionamientos.

No puede negarse la sana intención, pero progresar y encontrar obstáculos no impide atender asuntos por discernir y dilemas éticos por analizar. Es deseable la comunicación de testimonios para mejorar el trabajo del área operativa, y hasta pudiera invocar nueva teorización. Por lo pronto, la reacción inmediata tiende a subestimar e interpretarlos como trivialidades. Tales avisos importan, pues son evidencias de un hecho real y merece formalizarse con opiniones de peso.

Las anomalías se refieren a la comisión de errores de diagnóstico, no imputables directamente al médico que los aplica, pero que al ocurrir en la práctica médica y en varias latitudes del mundo, descalifica a la relación médico-paciente. No obstante, sucede que los lineamientos recomendados han sido fielmente acatados, cumplen con evitar las enfermedades, descubren portadores de riesgo, da oportunidad de tratarlos y se les previene, no sólo de las miserias en cada uno, sino que realmente aminora la demanda asistencial de la población ${ }^{5}$. Ciertamente, haría falta tener más testimonios para fortalecer una opinión, porque aisladamente el problema puede pasar incomprendido y cada actor justificaría deslindarse de esa responsabilidad. La situación es que realmente ocurre, y hay que ponerle atención.

Es paradójico que partiendo de una buena intención, buscando el beneficio para todos, esto sea el blanco de inconvenientes por haber generado diagnósticos equivocados, costos agravados, y que en conjunto, le reste credibilidad a la calidad, terminando por ser indeseable. El ámbito de esta antinomia se vive más con los pacientes o personas de la comunidad y las acciones públicas, que entre quienes han construido la teoría y diseñado las prácticas de programas $^{6}$. Al problema se le puede ubicar entre lo que no se sabe, por insuficiente información, de lo que ya es sólido conocimiento.

La esencia del problema puede comprenderse mejor al revisar el concepto de "pre-enfermedad" en los términos a que da lugar en la práctica, algunos de estos son: pre-infarto, pre-diabetes, pre-hipertensión, pre-canceroso, pre-obesidad, entre otros. Además, al usar el mismo criterio, no impide extenderlo a condiciones tales como pre-embarazo y pre-mortem, que resalta mejor el dislate y acentúa su insensatez. Aparte, los conceptos a los que aluden los términos son debatibles, favorecen la controversia, dan lugar a desacuerdos y hasta propician prácticas engañosas, no éticas, favoreciendo manipulaciones lucrativas, abusivas y engañosas. Es evidente que hay algo mal y es imperativo, por lo menos no fomentarlo y que siga teniendo objeto de análisis.

El concepto de "pre-enfermedad" es incongruente, grave y destructivo. No identifica con certidumbre, por ser producto del azar, añade confusión, tiende a ser indefinido en el tiempo y desorienta en cuanto al verdadero sentido de la enfermedad. Pese a que lleva la pretensión de determinar mejor a la condición o enfermedad, se opone al principio más valioso para identificarla: la certidumbre. El término 
debe haber resultado del uso sesgado, dando énfasis a la atención de riesgos posibles, pero ha resultado contra producente, empeora las cosas, está muy lejos de rendir precisión y exactitud, es alarmante y la reacción en la población repercute contra la relación médico-paciente, una delicada relación social afectada en los atributos que más le prestigia: "una confianza frente a una conciencia".

Parte de lo mismo es la aparente arbitrariedad para asignar valores numéricos a los "puntos de corte"'. Se entiende en ellos, la intención de establecer una línea de demarcación, misma que no existente entre las condiciones fisiológicas y las fisiopatológicas, por ser variables biológicas continuas. Sir George Pickering ${ }^{7}$ (1904-1980), retando a la audiencia del Real Colegio de Médicos de Londres, pedía que se dijera cuál es la presión arterial normal y cuál no, porque ésta tiene una distribución continua y unimodal. En sus palabras "es una falacia carente de evidencia biológica, separar a la población en dos grupos distintos y claramente definidos, los normotensos y los hipertensos".

A lo anterior, añádase que las cifras escogidas son sólo puntos de referencia en un parámetro de una duda cuantificada. Naturalmente que al variar los "puntos de corte", varían las estadísticas de prevalencia y de riesgos en la población, que también se ha usado falazmente. Al no tener esta contradicción, una explicación plausible, se define como absurda. Esto es lo que sucede al no aplicar los conceptos para lo que fueron diseñados desde un principio, en donde su utilidad es indiscutible.

Es obligado aclarar la confusión relacionada entre normalidad y riesgo. Riesgo es un concepto más allá de la normalidad, tienen enfoques completamente distintos y no comparables $^{8}$. Normalidad, deriva del lenguaje estadístico, considera a una población, con distribución central (tendencia al promedio), en la que se asume de antemano que domina la no-enfermedad. Sirve como puntos de referencia para juzgar como saludables a los parámetros biológicos. Según su distribución, la ubicación de los extremos es la no-saludable. En cambio riesgo, es un concepto que presupone una exposición a peligro en toda la población, en principio afecta a toda una comunidad. Entonces, la variable asociada es simplemente un indicador, un marcador, aunque en algunas circunstancias resulta coincidir con ser antecedente de enfermedad. Establece la regla de que a mayor valor del parámetro, mayor riesgo y a menor valor, menor riesgo. En esto no hay posible normalidad, de modo que son dos conceptos diferentes que sirven a fines completamente distintos. Por lo frecuente que ocurre, es recomendable no confundirlos. En la práctica la gente sigue preguntando ¿cuál es el colesterol normal?, ¿cuál la presión arterial? Y así sucesivamente con los ya acreditados factores de riesgo'. Por fortuna no ocurre lo mismo con estatura, color de piel o velocidad del automóvil, que también llevan riesgo inherente, y sin embargo nadie pregunta ¿cuál es lo normal?

Algo digno de tomarse en cuenta, de su amplia perspectiva, es el proceso que se sigue para conocer o reconocer a ENT. Se efectúa con los principios del conocimiento, basados en la verdad. Importan mucho por el peligro de caer en falacia. Ocurre no sólo en el ámbito de la medicina, sino en cualquier otra área del conocimiento y cultura, a donde intervenga el razonamiento. El Dr. José María Ménde ${ }^{10}$, de Madrid, España ha concentrado el tema en "La falacia más nefasta de la historia', aclarando que el paso en falso puede ocurrir en el lenguaje ordinario o en cualquier juicio, así sea identificar una enfermedad, pues es el conocimiento mismo.

Consiste en que se da por hecho verídico, lo que sólo parece serlo, o sólo da la impresión. Esto vale en la probabilidad de ser causa, riesgo o enfermedad. Por muy cercana que se estime a la verdad, probabilidad del 95\%, no lo es por completo. Estrictamente, no es permisible identificar sin más, que exista una enfermedad en una persona cuando pueda ser falsa. La expresión de "pre-enfermedad" no puede significar a la enfermedad, aunque fuera muy cercana la estimación de ser cierta. Consecuentemente, en una población estudiada siempre surgirá la falsamente considerada. Es prudente entonces, no dejar de verificar el caso, cerciorarse de que es verdad o por lo menos llegar a la evidencia, que en esto el buen criterio clínico desarrolla su arte humanizante.

Dado que esto pasa de la simplicidad a la complejidad, lo acertado es revisar todo aquello que puede estar involucrado para no caer en falacia. Los testimonios desde el punto de vista médico ocurren análogamente en justicia, a donde se dice que no hay ciencia para casos concretos. Así, la dinámica del avance cognitivo, unas veces en lo teórico y otras en lo prácticos, presiona a comprender mejor los cambios y hacer adaptaciones del entorno. El desenlace cambiará al aplicar sabios ajustes adaptativos.

En conclusión, el concepto de "pre-enfermedad" simplemente no es necesario, ni suficiente para añadir atributos en la identificación de una condición o enfermedad. Los "puntos de corte"' hay que tomarlos como lo que son: parte de la estandarización metodológica, prácticos para orientar su propósito, definiciones operativas y no conceptuales. Su uso tiene connotaciones diferentes según el campo de aplicación, que difieren entre la clínica y la salud pública.

Al tratar de enmendar problemas, es recomendable evitar el reduccionismo propio de las especialidades, que no sólo crea términos nuevos, sino que usa los conocidos con otros conceptos, favorece los malos entendidos y la falacia. De esta manera, no hay comunicación entre expertos y bastaría buena disposición de multidisciplinariedad para dar oportunidad a la conciliación y zanjar brechas del conocimiento. Buena parte de los problemas en las áreas de la salud terminarían con orientarse por medio de la educación continua, y con la moderna óptica de las Ciencias de la Complejidad ${ }^{11}$ al alcance de todos.

\section{Referencias}

1. Cardenas M. Dislates en los escritos médicos. Arch Cardiol Mex 2010;4:315-7.

2. Blackburn $\mathrm{H}$. Ischaemic heart disease: Perspective for prevention. Heart Bull 1971;2:94-7.

3. Rose GA, Blackburn H, Gillum RF, et al. Métodos de encuesta sobre enfermedades cardiovasculares. Ginebra: Organización Mundial de la Salud; 1982.

4. Hill AB. Statistical Methods in Clinical and Preventive Medicine. Livingstone, Edinburgh: Churchill Livingstone; 1962.

5. Koepsell TD, Wagner EH, Cheadle AC, et al. Algunos aspectos metodológicos de la evaluación de los programas de promoción de la salud y prevención de las enfermedades basados en la comunidad. En: Organización Mundial de la Salud. Promoción de la salud: una antología. Washington: Organización Mundial de la Salud; 1996. p. 209. 
6. Organización Panamericana de la Salud. Promoción de la Salud: una antología. Washington: Publicación Científica No. 557 OMS; 1996.

7. Pickering G. Nature of essential hypertension. London: Churchill Livingstone; 1981.

8. Galen RS, Gambino SR. Beyond Normality. En: The predictive value and efficiency of medical diagnoses. New York: John Wiley \& Sons; 1975.

9. Berkeley G. Principios del conocimiento humano. México: Gernika; 1994. p. 14.

10. Méndez JM. La falacia más nefasta de la historia. Estudios de Axiología. Madrid 2010:1-14.

11. McLaren P. Multiculturismo Crítico. Säo Paulo: Cortez Editora; 1997.
Rafael César Chávez-Domínguez*

Departamento de Epidemiología, Instituto Nacional de Cardiología Ignacio Chávez, México D.F., México

* Autor para correspondencia: Instituto Nacional de Cardiología Ignacio Chávez. Juan Badiano 1, Colonia Sección XVI, Tlalpan. C.P. 14050. México D.F., México.

Tel.: +011 (52) 55732911.

Correo electrónico: chavezrafael@hotmail.com

http://dx.doi.org/10.1016/j.acmx.2012.06.002 